\title{
Your conflict matters to me! Behavioral and neural manifestations of control adjustment after self-experienced and observed decision-conflict
}

\author{
Jasper Winkel 1,2*, Jasper G. Wijnen', K. Richard Ridderinkhof ${ }^{1}$, Iris I. A. Groen ${ }^{1}$, Jan Derrfuss ${ }^{3}$, \\ Claudia Danielmeier ${ }^{3}$ and Birte U. Forstmann ${ }^{1,2}$
}

\begin{abstract}
Amsterdam Center for the Study of Adaptive Control in Brain and Behavior, Department of Psychology, University of Amsterdam, Amsterdam, The Netherlands
2 Spinoza Center, University of Amsterdam, Amsterdam, The Netherlands

${ }^{3}$ Max Planck Institute for Neurological Research, Cologne, Germany
\end{abstract}

Edited by:

Francisco Barceló, University of Illes

Balears, Spain

Reviewed by:

Jin Fan, Mount Sinai School of

Medicine, USA

Hong Li, Southwest University, China

*Correspondence:

Jasper Winkel, Department of

Psychology, University of Amsterdam,

Roetersstraat 15, 1018WB,

Amsterdam, The Netherlands.

e-mail: jasperwinkel@gmail.com
In everyday life we tune our behavior to a rapidly changing environment as well as to the behavior of others. The behavioral and neural underpinnings of such adaptive mechanisms are the focus of the present study. In a social version of a prototypical interference task we investigated whether trial-to-trial adjustments are comparable when experiencing conflicting action tendencies ourselves, or simulate such conflicts when observing another player performing the task. Using behavioral and neural measures by means of event-related brain potentials we showed that both own as well as observed conflict result in comparable trial-to-trial adjustments. These adjustments are found in the efficiency of behavioral adjustments, and in the amplitude of an event-related potential in the N2 time window. In sum, in both behavioral and neural terms, we adapt to conflicts happening to others just as if they happened to ourselves.

Keywords: social cognition, Simon task, conflict adaptation, Gratton effect, observed conflict, event related potentials, N2

\section{INTRODUCTION}

In our daily lives, we need to selectively control and adjust our behavior. Wrong choices may instantly appeal to us, and yet we override these in order to respond based on what we have learned is right. This is indicative of how our brain selects responses: automatic bottom-up processes capture our action system, based on salient stimulus properties or strong impulses, and top-down action control is applied, based on intentions, instructions, and previous experience. Understanding the actions of others may be central to the human condition. Evolutionary thinkers believe that much of the improved cognitive abilities that humans and other apes possess, originate from the adaptive advantage of understanding and predicting the cognition and behavior of our peers; the Machiavellian intelligence hypotheses (Byrne and Whiten, 1988; Whiten and Byrne, 1997). Here we ask what happens if the previous experience that lead us to implement control is not our own behaviour, but rather derived from performance observed in another. And more specifically, how do we tune our behavior to that of others? This study set out to answer these questions by zooming in on trial-by-trial adjustments using a social interference task.

Trial-to-trial adjustments have been studied with different interference tasks such as the Stroop task, the Eriksen flanker task, and stimulus-response compatibility tasks such as the Simon task (e.g., Pardo et al., 1990; Hazeltine et al., 2003; Schumacher et al., 2007). In such tasks, responses are typically slowed on incongruent (I) trials, that is, when the relevant aspect of the stimulus requires a response opposite to the response triggered by task-irrelevant stimulus aspects, rather than when the activated responses overlap, as on congruent (C) trials. The magnitude of the behavioral interference effect of congruence (i.e., I trials-C trials) is often considered as a measure for the ability to resolve response interference within a trial which may involve selective inhibition of the automatic response to reduce interference between competing actions (Simon and Wolf, 1963; Simon and Rudell, 1967; Forstmann et al., 2008a,b). Interestingly, when zooming in on trial-by-trial adjustments, it becomes evident that the interference effect is reduced after I compared to C trials (Gratton et al., 1992). This finding refers to the so-called conflict adaptation effect, or Gratton effect, which can be quantified as the difference of the interference effect following incongruent trials (iI-iC), subtracted from the interference effect following congruent trials $(\mathrm{cI}-\mathrm{cC})$. For brevity, in the following text we will use a lower case to denote the previous trial and an upper case to denote the current trial.

An influential theory capturing the conflict adaptation effect is the conflict monitoring hypothesis (Botvinick et al., 1999, 2001, 2004; but see also Mansouri et al., 2009). It proposes that in order to resolve response conflict, the presence of this conflict must first be detected and evaluated. Subsequently, cognitive control is implemented in order to overcome the detected conflict. Once control processes are activated they can also act on the following trial: when the subsequent trial is again incongruent, the already implemented control processes lead to an advantage in overcoming conflict. Hence, faster reaction times (RTs) are observed on iI trials compared to cI trials which results in an overall reduction of the interference effect after I trials compared to $\mathrm{C}$ trials.

The activation of conflicting response tendencies is typically accompanied by activation of posterior brain areas within the medial frontal cortex, most prominently the rostral cingulate zone (RCZ; for review see Ridderinkhof et al., 2004a). Selecting the taskappropriate action is more demanding when competing alternative actions are activated on the basis of task-irrelevant stimulus 
features. Such processes of action selection are typically associated with activation in dorsal brain areas within the medial frontal cortex, most prominently the pre-supplementary motor area (preSMA; for review see Nachev et al., 2008). Under more demanding circumstances, additional activation is observed in lateral prefrontal cortex (LPFC; for review see Mostofsky and Simmonds, 2008). Studies using functional magnetic resonance imaging to examine the Gratton effect have shown that RCZ activation associated with conflicting response tendencies is followed by activation in LPFC (e.g., Kerns et al., 2004; Kerns, 2006), presumably as a means of increased proactive top-down guidance of action selection processes after conflict has been experienced, such that further response capture by task-irrelevant stimulus features is pre-empted.

Action selection processes, especially when facing conflicting response tendencies, are expressed in event-related brain potentials (ERPs) obtained from frontocentral scalp sites. Although it is not always clear whether these manifestations reflect activation in preSMA or in the directly underlying RCZ, frontocentral ERPs are consistently modulated by factors that place differential demands on action selection, most typically in the time window of the so-called N2 (for review see Folstein and van Petten, 2008). Many studies have examined these modulations in the context of conflicts tasks, expressed in a negative shift in the frontocentral ERPs in the N2 time range (e.g., Heil et al., 2000). This negative shift is modulated by the Gratton effect, such that the negative shift was considerable after congruent trials but much reduced after incongruent trials, mirroring the RT results (Leuthold and Schröter, 2006). This pattern again suggests pre-emptive control after experienced conflict, such that action selection is subsequently less affected by response capture from task-irrelevant stimulus features.

In the present study, we introduce a social version of the Simon task, where during half of the trials, the subjects observe another person's response instead of responding themselves. This allows us to examine trial-by-trial adjustments, after having observed behavior of someone else compared to the adjustments after performing the task oneself. According to the simulation account (Gallese et al., 2004; Ramnani and Miall, 2004; Iacoboni et al., 2005; Decety and Grèzes, 2006) the observation of another person's behavior induces internal states in the observer that are similar to those that would occur if the observer undertook the action himself. Interestingly, these internal states are also evoked if the other's actions are not directly observed, but heard or suggested instead (Umiltá et al., 2001; Kohler et al., 2002). These findings suggest that it is not simply perceiving an action, but knowing that it takes place, that evokes these states. As such, we expect that observing the Simon task in another will also evoke response conflict in the observer. Therefore, we examine whether the Gratton effect as obtained after self-experienced response conflict is also obtained after observing the performance of someone else.

Two hypotheses are formulated: first, we expect to find comparable trial-to-trial adjustments in the shape of a behavioral Gratton effect after the observation of someone else or after performing oneself. Second, we expect the negative shift in the frontocentral ERPs in the N2 time range to be modulated by the Gratton effect, such that a negative shift is observed after congruent but not incongruent trials. Again, this modulation should be comparable after having observed someone else compared to having performed the task oneself on the previous trial.

\section{MATERIALS AND METHODS PARTICIPANTS}

Twenty healthy volunteers (22-28 years old, $\mu=24, \sigma=1.65$ ) were recruited from the student population of the University of Amsterdam. The subjects were all right handed as assessed by the Edinburgh Inventory (Oldfield, 1971). Informed consent was obtained prior to the experiment. The experiment was approved by the local ethics committee of the University of Amsterdam, and all procedures were conducted in accordance with relevant laws and institutional guidelines. All subjects had normal or corrected-tonormal vision. Subject reported having no history of neurological, major medical, or psychiatric disorder. Due to an error in the computer setup, no response markers were introduced in the EEG datafiles during the testing of five subjects. As a result, only the behavioral data from these subjects are reported. An additional behavioral session was acquired for 17 of the 20 subjects.

\section{PROCEDURE}

\section{Instructions}

Subjects were instructed that they were to perform in a task that was interspersed with trials previously recorded from another person performing in the same task. In reality, they observed computer generated behavior. The subjects were shown a picture and were given a name of the fictional other subject. Names of fictional subjects were matched in length to the names of the subjects participating in the experiment. Note that subjects were explicitly informed about the non-competitive nature of the experiment. Moreover, subjects were instructed not to respond during the other's trials, unless the other made an error. The latter trials served as catch trials. Before the experiment started the subjects were also photographed to increase the plausibility that their own data would be used as an 'other' for a future subject. Subjects had already performed this task for $40 \mathrm{~min}$ previously, and subjects were allowed to practice the task for $10 \mathrm{~min}$ before the experiment began.

\section{Debriefing and questionnaire}

After performing the experimental session, subjects filled out a visual analog scale questionnaire. The questions were set up to probe the subjects' representation of the other player, without directly asking their belief in the manipulation. Once the subjects had filled out this questionnaire, they were informed about the deception, and asked directly whether they had believed it. Their answer to this final question was rated on a 5-point scale, which was included in the overall measure of the strength of the subjects' representation of the other, ranging from 0 to 1 .

\section{Behavioral task}

Subjects performed a social version of the Simon task (Simon and Wolf, 1963) with four blocks of 320 trials each. Throughout the experiment, two buttons were visualized in the lower left and lower right of the screen, a visual angle of $5.6^{\circ}$ apart (see Figure 1). A white central fixation cross was presented on a black background for $1000 \mathrm{~ms}$, followed by a $500 \mathrm{~ms}$ cue (the subject's name or the other), indicating who was to perform the upcoming trial. This cue was followed by a fixation cross for $800 \mathrm{~ms}$, and then the target appeared, lasting for $1000 \mathrm{~ms}$. Targets were filled circles of one of four colors (red and green for a right response, blue and yellow for 


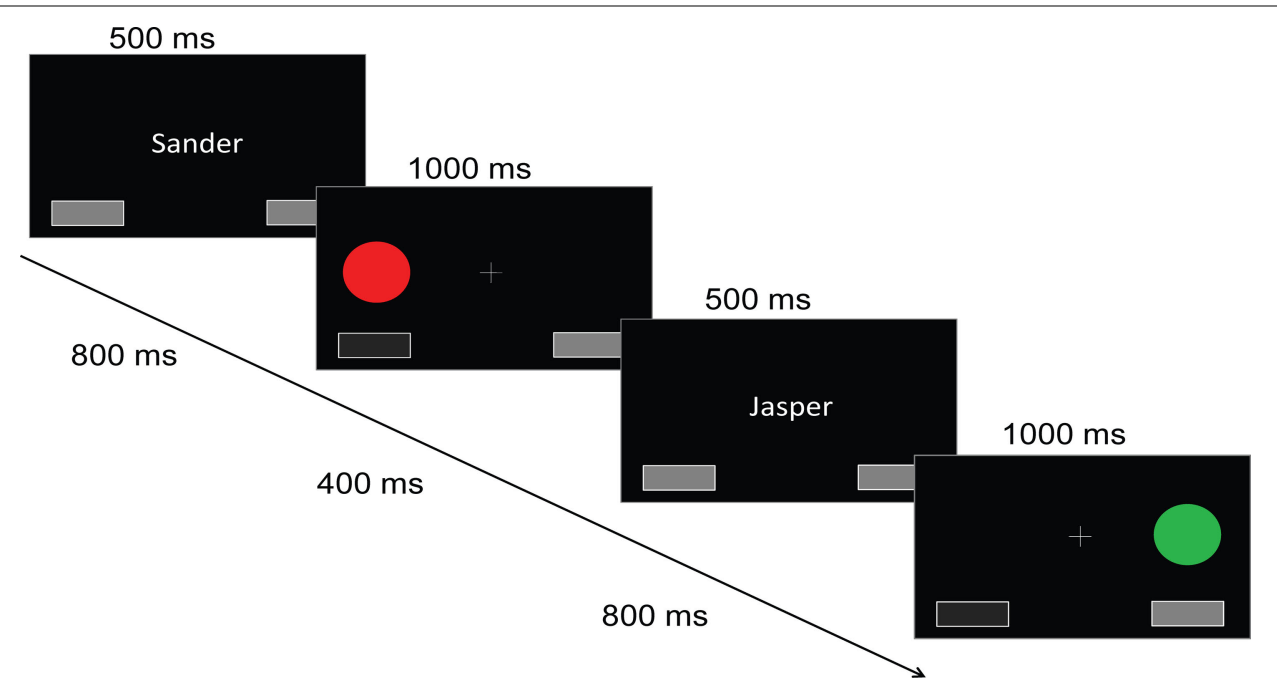

FIGURE 1 | Social Simon task. Schematical drawing of the social Simon task Each trial started with the presentation of a name cue. In the self condition, participants saw their own name presented on the middle of the screen. In the other condition, participants received a name of someone else. This name signaled that participants had to observe the behavior of this person and were not required to press a response button when the Simon stimuli were presented. In both the self and the other condition, participants viewed two response panels that were presented below the colored Simon stimuli. According to the response button that was pressed by the participants themselves, the spatially-compatible visually presented response panel was highlighted. In the other condition, one of the visually presented response panels was also highlighted indicating the other persons' response. Four different colored circles were used that were presented either on the right or left side of the central fixation cross, respectively. Green and red circles were mapped to a left response button press whereas yellow and blue circles were mapped to a right response button press. There were either congruent, i.e. color and spatial location overlap, or incongruent conditions, i.e. color or spatial location do not overlap. No stimulus repetitions were allowed. Cue-trial and inter-trial intervals are not depicted for sake of clarity. a left response). Targets appeared to the left or right of fixation, above either the left or the right button. The distance between the fixation cross and the target covered a visual angle of $2.8^{\circ}$. Responses were either generated by the computer, or made by the subject using response buttons, depending on the trial type (self or other). The selected response was visualized by pressing and depressing the corresponding button on the screen. After a delay of $400 \mathrm{~ms}$, a new trial began.

The sequence of trial conditions was produced randomly, with several constraints: the subject's trials comprised eight types $(2 \times 2 \times 2)$, as determined by the player of the previous trial (s/o), the congruency of the previous trial (c/i), and the congruency of the current trial $(\mathrm{C} / \mathrm{I})$. The experiment was set up so that each of the eight resulting combinations occurred equally often. Also, consecutive trials never used the same color, to avoid stimulus repetition effects and hence precluding an interpretation of the Gratton effect in terms of repetition priming (e.g., Wühr and Ansorge, 2005). To allow for this, two colors were mapped to each response direction. The other's RT was drawn from a uniform distribution between 220 and $460 \mathrm{~ms}$.

Moreover, six catch trials were included per block which ensured the subjects' attention during the other's trials as well as a high ecological validity. A third response button (under the middle finger of the right hand) was dedicated to responding to these catch trials.

\section{DATA ANALYSIS}

\section{Behavior}

For both the RT and the electrophysiological analyses, we removed both the error and catch trials, as well as the trials immediately following these. The remaining trials were categorized into one of eight categories (scC, scI, siC, siI, ocC, ocI, oiC, oiI). Note that in all of these conditions, the subject performs the task, and that it is only the self/other condition of the previous trial that varies. The Gratton effect was determined as the interaction effect between the congruency of the current trial and the congruency on the previous trial. Error rates were also computed for each of these eight trial types. RT and error rates were analyzed using SPSS 17 (SPSS Inc.).

\section{Electrophysiology}

Electrophysiological data was recorded using the 'Biosemi active two' acquisition system (Biosemi B.V., the Netherlands), recording from 32 scalp electrodes placed according to the 10/20 system (Jasper, 1958). Additionally, horizontal and vertical eye movements were recorded, next to the canthus of the left and right eye, and above and below the left eye. An electrode placed on the left earlobe was used as a reference signal.

The data was analyzed using Brain Vision Analyzer (v.1.05.0005, Brain Products $\mathrm{GmbH}$, Germany). The raw data was downsampled to $512 \mathrm{~Hz}$. The data were initially band-pass filtered with a lower cut-off of $0.5 \mathrm{~Hz}$ (12 dB/octave) and an upper cut-off of $20 \mathrm{~Hz}$ (48 dB/octave). Any noisy segments were located by visual inspection and removed. Eye-movement artifacts were isolated using Independent Component Analysis, and subsequently removed from the data (Vigario, 1997; Hyvarinen and Oja, 2000; Iriarte et al., 2003; Wijnen and Ridderinkhof, 2009). ERPs were aligned to a baseline of the $200 \mathrm{~ms}$ prior to target onset $(t=0 \mathrm{~ms})$. The data were exported from Brain Vision Analyzer, and the values for $\mathrm{Fz}$ and $\mathrm{Cz}$ were analyzed using SPSS.

Frontocentral ERPs in the N2 time window (between 200 and $400 \mathrm{~ms}$ ) were expected to show greater negativity in cI compared to $\mathrm{cC}$ trials. We identified such a shift based on the difference waves 
of cI and cC. This negative shift occurred between 270 and $400 \mathrm{~ms}$, and peaked at $328 \mathrm{~ms}$ (see Figure 3). We selected the area between 300 and $350 \mathrm{~ms}$ to zoom into this timespan. Adopting a procedure previously used in a number of similar studies (Kopp et al., 1996; Heil et al., 2000; Bartholow et al., 2005; Leuthold and Schröter, 2006) we analyzed the interval by computing the average voltage over the timespan.

The P3 component of the ERP follows the N2. Because the P3 spans a wide time interval and shows a broad scalp distribution, it shows partial overlap with the N2 both temporally and spatially (Nieuwenhuis et al., 2003; Yeung et al., 2004). In order to minimize the effects of this overlap on our analyses, we also filtered the data again to exclude the slow P3 component, using a band-pass filter with a lower cut-off of $3.5 \mathrm{~Hz}$ (12 dB/octave) and an upper cut-off of $20 \mathrm{~Hz}$ (48 dB/octave), (Donkers and van Boxtel, 2004; Wijnen and Ridderinkhof, 2009). Afterwards, we repeated our analysis of the negativity. We also computed the highest negative peak between 200 and $400 \mathrm{~ms}$ on the individual $\mathrm{cI}-\mathrm{cC}$ difference waves, to acquire an individual measure of the latency of the ERP negativity.

\section{Bayesian analysis}

In the remainder of the statistical analyses, we report Bayesian posterior probabilities in addition to conventional $p$-values on the ERP and the behavioral data to show that the effects for self and for other were identical. When we assume, for fairness, that the null hypothesis and the alternative hypothesis are equally plausible a priori, a default Bayesian $t$-test (Wetzels et al., 2009) allows one to determine the posterior plausibility of the null hypothesis and the alternative hypothesis. We denote the posterior probability for the null hypothesis as $p^{\text {Bayes }}\left(H_{0}\right)$. When, for example, $p^{\text {Bayes }}\left(H_{0}\right)=0.9$, this means that the plausibility for the null hypothesis has increased from 0.5 to 0.9 , and the plausibility of the alternative hypothesis has correspondingly decreased from 0.5 to 0.1 . We report these posterior probabilities because they address several problems both with conventional $p$-values and with $p_{\text {rep }}$ (Wagenmakers, 2007; Iverson et al., 2008a,b). Most importantly, posterior probabilities allow one to directly quantify evidence in favor of the null hypothesis, instead of only 'failing to reject' it. In the case of our analyses, we perform a one-sample Bayesian $t$-test on the difference scores of two measures (following self and following other), because we want to show the posterior probability that they are the same. This relates directly to our hypotheses following the simulation account, proposing that the same behavioral and neural modulations occur following own, and following observed behavior.

\section{RESULTS \\ BEHAVIORAL RESULTS}

Average RT was $572 \mathrm{~ms}(\sigma=42)$. Average ER was $0.037(\sigma=0.023)$. The average strength subjects' representation of the other was $0.54(\sigma=0.17)$.

We performed two repeated-measures ANOVAs, one with RT as the dependent variable, and one with error rate as the dependent variable. Both analyses used previous congruency, current congruency, and previous self/other condition as independent variables.

The only significant result was found for the interaction effect between previous congruency and current congruency on RT, with a larger Simon effect following congruent, than following incongruent trials $[F(1,19)=6.147, p=0.023]$ (See Figure 2A).

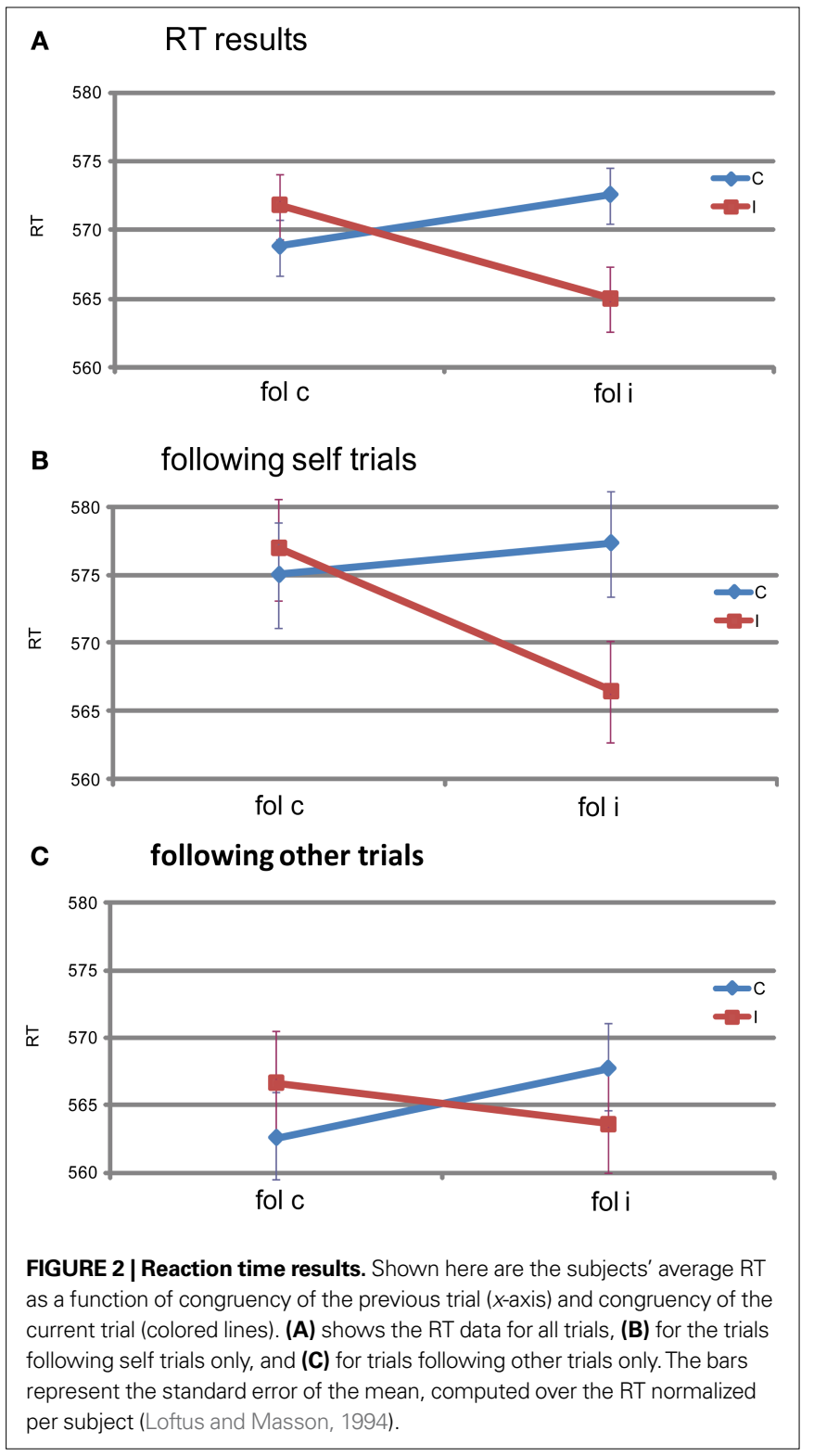

There was a marginal main effect of previous congruency on error rates $[F(1,19)=3.924, p=0.062]$, with error rates being higher following incongruent $(0.049)$ than following congruent trials (0.043).

When split for following self and following other trials, the interaction effect on RT between current and previous congruency was marginally significant following self $[F(1,19)=3.67, p=0.070]$, and marginally significant following other $[F(1,19)=3.07, p=0.096]$. The directions of these two interactions are the same (Figures 2B,C). Bayesian analysis on the size of the Gratton effect following self and following other trials revealed that there was no difference between the two $\left[t(19)=0.595, p=0.559, p^{\text {Bayes }}\left(H_{0}\right)=0.83\right]$. No other tests revealed significant effects $(F<1)$.

Note that no main effects of current congruency on RT or ER (i.e. Simon effects) were found in our behavioral data. However, we find that the size of the Simon effect during our experiment 
and during the previously recorded behavioral session using the same paradigm show a significant correlation $[R(17)=0.584$, $p=0.007$ (one-sided)].

\section{EEG RESULTS}

Visual inspection of frontocentral ERPs suggests that, as expected, I trials were associated with a greater negativity in the $\mathrm{N} 2$ time window (between 200 and $400 \mathrm{~ms}$ ) than C trials (see Figure 3A). Modulations of this effect by the congruity of the preceding trial were examined by determining area averages in the difference waves $\mathrm{cI}-\mathrm{cC}$ versus $\mathrm{iI}-\mathrm{iC}$. A substantial negative shift occurred in the cI-cC difference wave between 270 and $400 \mathrm{~ms}$, peaking at $328 \mathrm{~ms}$. Voltage maps confirmed the frontocentral scalp distribution of this negative shift (see Figure 3C). Notably, such a negative shift was conspicuously absent in the iI-iC difference wave. Repeatedmeasures ANOVAs on the average EEG amplitude between 300 and 350 ms were computed separately for the electrodes of interest, $\mathrm{Fz}$ and $\mathrm{Cz}$. The average amplitude of the negative shift at $\mathrm{Fz}$ shows a significant interaction effect between current congruency and previous congruency $[F(1,14)=9.962, p=0.007]$ (see Figure 3B). The average amplitude of $\mathrm{Cz}$ shows a similar Gratton effect $[F(1,14)=6.175, p=0.026]$. P2 amplitudes showed no significant effects (all $p>0.1$ ).

The same analysis was repeated for ERPs with the P3 filtered out. The results were comparable, and more reliable. The average amplitude of $\mathrm{Fz}$ shows a significant interaction effect between current congruency and previous congruency $[F(1,14)=17.375$, $p=0.001]$, as does the average amplitude of $\mathrm{Cz}[F(1,14)=12.189$, $p=0.004]$.

The interaction effect on $\mathrm{Fz}$ was significant in the trials following self trials $[F(1,14)=6.527, p=0.023]$, and in trials following other trials $[F(1,14)=6.232, p=0.026]$. The interaction effect on $\mathrm{Cz}$ was also significant in the trials following self trials $[F(1,14)=4.960$, $p=0.043]$, and in trials following other trials $[F(1,14)=4.638$, $p=0.049]$. Bayesian analysis revealed that the $\mathrm{N} 2$ effect across the four trial types were identical for previous self and previous other on both $\mathrm{Fz}\left[t(14)=0.19, p=0.86, p^{\text {Bayes }}\left(H_{0}\right)=0.83\right]$ and $\mathrm{Cz}$ $\left[t(14)=-0.38, p=0.71, p^{\text {Bayes }}\left(H_{0}\right)=0.83\right]$. Also, there is no difference between the interaction effect following self and following other trials for $\mathrm{Fz}\left[t(14)=-0.6, p=0.55, p^{\text {Bayes }}\left(H_{0}\right)=0.81\right]$, and $\mathrm{Cz}\left[t(14)=-0.64, p=0.53, p^{\text {Bayes }}\left(H_{0}\right)=0.81\right]$.

The average latency of the individual maximum difference between $\mathrm{cI}-\mathrm{cC}$ was $341 \mathrm{~ms}(\sigma=33 \mathrm{~ms})$. These latencies showed a significant positive correlation with individual RTs $[R(15)=0.597$, $p=0.018$ (two-tailed) .

\section{DISCUSSION}

The present study set out to investigate adaptive control mechanisms based on observed behavior. More specifically, using a Simon task, we measured the Gratton effect, which is a modulation of the interference effect of the current trial by the congruency condition of the previous trial. According to the simulation account, subjects attempt to understand the behavior of others by utilizing their own neural circuitry to simulate the mental processing underlying that behavior. Such a view has been firmly established in the domain of motor actions, but several studies have extended it to cognitive processing (van Schie et al., 2004; Schuch and Tipper,
2007; Tsai et al., 2008). Based upon the simulation account, we hypothesized that we would observe a similar Gratton effect following a subject's own conflict, and following the observation of conflict in another.

Most importantly, our results show a similar Gratton effect following performed, and observed conflict. This is in line with our hypothesis that subjects implement lasting control when observing a conflict situation in another's behavior. The presence of control in subjects' own behavior after an observed incongruent trial suggests that subjects also simulate conflict during that observed trial. This finding suggests that when we observe someone else performing a conflicting task, we experience conflict ourselves. The fact that we find frontocentral negative shifts in the ERP, which match the Gratton effect on RT supports this notion and indicates that difficulties in action selection, as triggered by salient but task-irrelevant stimulus features, are pre-empted by response conflict in the previous trial, regardless of whether that conflict was self-experienced or simulated based on the observed performance of someone else.

The Gratton effect we observed on RT did not retain statistical significance when split for self and other. However, we did find marginal significance in both these split conditions, and the absence of a three-way interaction effect between self/other, previous, and current congruency suggests that our significant Gratton effect was not specifically driven by only the following self, or only the following other trials. Further support for this notion is provided by our Bayesian analysis. We found high posterior Bayesian probability, showing that the effects following self and following other were the same. Therefore, we suggest that the Gratton effects following self and following other trials are similar.

At first glance, the present data seem to be inconsistent with previous findings. First, we did not obtain a Simon effect on either RT or error rates. Second, compared to previous ERP results, our frontocentral negativity occurs slightly later than the N2 peak normally found in the Simon task. One explanation for the aforementioned discrepancies might be the overall prolonged RTs as compared to classical Simon tasks. In our experiment, the mean RT was $572 \mathrm{~ms}$ whereas in classical Simon tasks mean RTs are much shorter, e.g., $473 \mathrm{~ms}$ (Hommel, 1994); $485 \mathrm{~ms}$ (Notebaert et al., 2001), or $496 \mathrm{~ms}$ (Hommel et al., 2004). As such, responses in our modified experiment were $75-100 \mathrm{~ms}$ slower than in the basic Simon task. Previous experiments have shown that increasing the delay between stimulus presentation and response decreases the Simon effect (Simon et al., 1976). Another study has demonstrated that increasing the complexity of the stimulus used in the Simon tasks results in longer RTs, as well as a complete abolishment of the Simon effect (Hommel, 1994). Increasing the size of the stimulus set mapped to each response hand also increases RT and decreases the overall Simon effect (Hommel, 1995). Note that an increased stimulus set size is also present in our experiment, because we use two colors mapped to each response hand. Further evidence for the phenomenon of decreased Simon effects with slower responses is found in research looking into RT distributions. In these types of analyses, the size of the interference effect is determined separately across different Vincentized time bins (De Jong et al., 1994). The results typically show strong Simon effects in fast response bins, and progressively smaller, even negative Simon effects in slower response bins (Burle et al., 2002; Ridderinkhof et al., 2004b). 


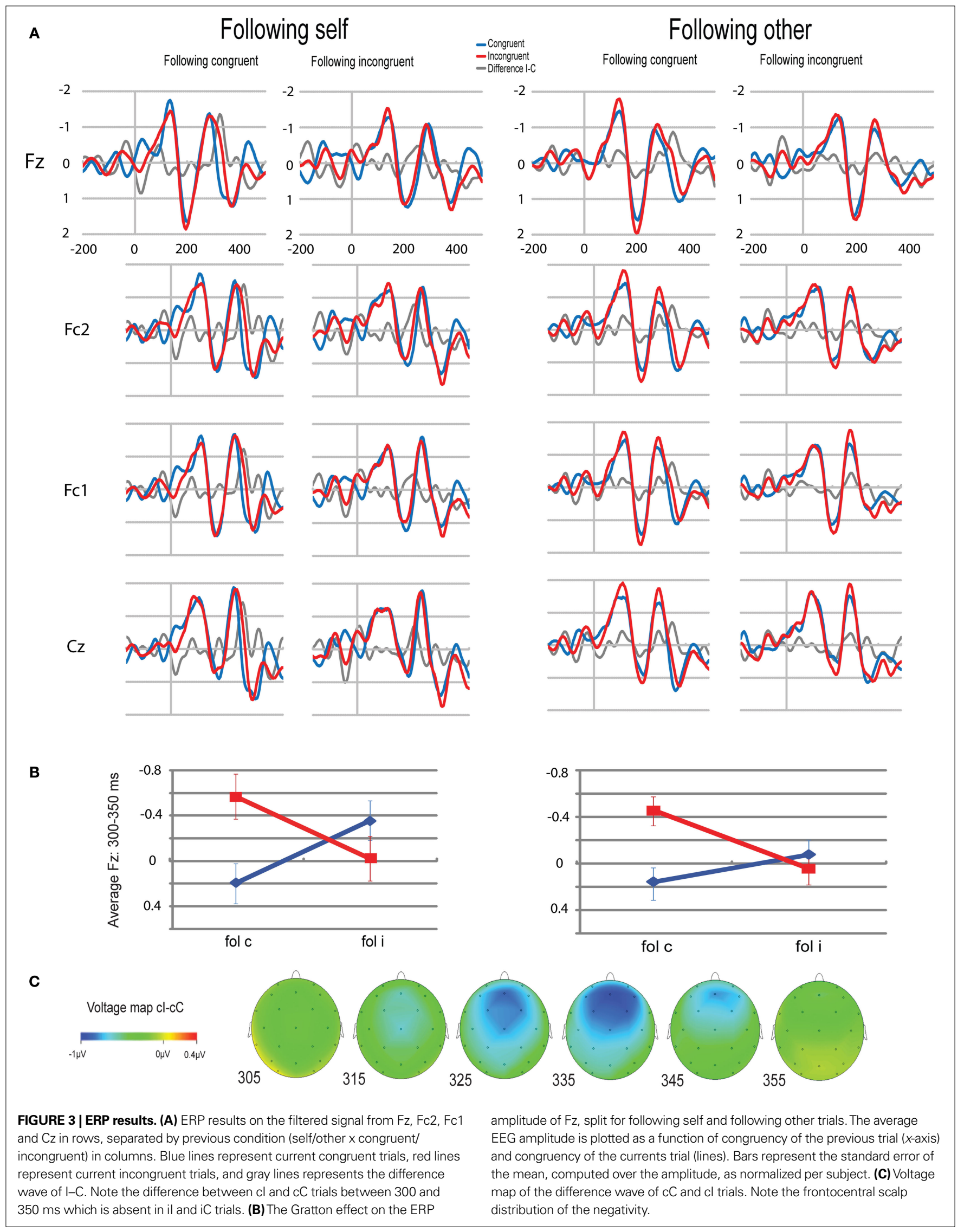


Negative Simon effects also occur following incongruent trials in several experimental manipulations, resulting in a net Simon effect of 0 in these conditions (Stürmer et al., 2002). This demonstrates that the reliability of the Gratton effect does not depend on the presence of a Simon effect.

We believe that such an increase in RTs might also account for the present results in that it caused participants to respond more slowly, thereby evading the early response capture process. Less response capture would also explain the missing Simon effect on error rates since most incongruent errors are committed during very fast responses. Interestingly, although the Simon effect is abolished in our data, the Gratton effect is maintained.

In addition to the behavioral consequences, the slower responses may also explain why the frontocentral negativity in the ERP is delayed compared to the commonly reported N2 peak. We propose that the frontocentral negativity might reflect the same processes underlying the $\mathrm{N} 2$ component; however, these processes are delayed due to complexity of the task. This results in a modulation of the slope between N2 and P3 instead. To support this notion, we analyzed the latency of our ERP effect on the subject-specific level by applying peak detection on subject-specific cI-cC difference waves. We found that the latency of subjects' frontocentral negativity correlated positively with their mean RT. This finding corroborates the idea that the latency of the frontocentral negativity is dependent on mean RT.

Our results complement other recent findings in social cognitive control. For instance, subjects who perform half a Simon task (by using only one response button) show an interference effect when they believe the other button is operated by a human, but not when they believe it is operated by a computer (Tsai et al., 2008). Subjects also show an error-related negativity when observing another's errors (van Schie et al., 2004), and afterwards they also show post-

\section{REFERENCES}

Bartholow, B. D., Pearson, M. A., Dickter, C. L., Sher, K. J., Fabiani, M., and Gratton, G. (2005). Strategic control and medial frontal negativity: beyond errors and response conflict. Psychophysiology 42, 33-42.

Botvinick, M., Nystrom, L. E., Fissell, K., Carter, C. S., and Cohen, J. D. (1999). Confict monitoring versus selection for-action in anterior cingulate cortex. Nature 402, 179-181.

Botvinick, M. M., Braver, T., Barch, D. M., Carter, C., and Cohen, J. D. (2001). Conflict monitoring and cognitive control. Psychol. Rev. 108, 624-652.

Botvinick, M. M., Cohen, J. D., and Carter, C. S. (2004). Conflict monitoring and anterior cingulate cortex: an update. Trends Cogn. Sci. (Regul. Ed.) 8, 539-564.

Burle, B., Possamaï, C. A., Vidal, F., Bonnet, M., and Hasbroucq, T. (2002). Executive control in the Simon effect: an electromyographic and distributional analysis. Psychol. Res. 66, 324-336.

Byrne, R. W., and Whiten, A. (1988). Machiavellian Intelligence. Social Expertise and the Evolution of Intellect error slowing (Schuch and Tipper, 2007). Our results and the results mentioned above can be considered to form a cognitive version of the simulation account, which proposes that we understand the cognitive processes of others by simulating them; generating a representation similar to that we would use ourselves (Gallese et al., 2004; Ramnani and Miall, 2004; Iacoboni et al., 2005; Decety and Grèzes, 2006). This hypothesis is based upon the discovery of the mirror neuron system (Di Pellegrino et al., 1992; Gallese et al., 1996), and the simulation account has been firmly established in the visuomotor domain. The system is thought to serve both to understand the actions of others, and likely also to facilitate learning through imitation (Rizzolatti, 2005). Research outside the motor domain indicates that the simulation account also holds for emotional states, such as disgust (Phillips et al., 1997; Calder et al., 2000; Small et al., 2003; see Gallese et al., 2004, for a review). According to the simulation account, in order to understand observed behavior, we represent observed actions as if they are our own. The finding that we adapt our subsequent behavior in the form of the Gratton effect indicates that this is also the case for observed conflict. Combined with the aforementioned results, this leads us to conclude that the simulation account also applies to cognitive control.

To summarize, the use of our novel paradigm has allowed us to show the existence of sustained control processes in the brain and behavior of the observer of an action. These results indicate that we represent observed conflict as if it were our own.

\section{ACKNOWLEDGMENTS}

This work was supported by a VENI grant (B.U.F.) and VICI grant (K.R.R.) from the Netherlands Organization for Scientific Research (NWO). We thank R. Wetzels and E.-J. Wagenmakers for valuable comments on the Bayesian statistics. in Monkeys, Apes, and Humans. Oxford, Clarendon Press.

Calder, A. J., Keane, J., Manes, F., Antoun, N., and Young, A. W. (2000). Impaired recognition and experience of disgust following brain injury. Nat. Neurosci. 3, 1077-1078.

Decety, J., and Grèzes, J. (2006). The power of simulation: imagining one's own and other's behavior. Brain Res. 1079, 4-14.

De Jong, R., Liang, C.-C., and Lauber, E. (1994). Conditional and unconditional automaticity: a dual process model of effects of spatial stimulus-response correspondence. J. Exp. Psychol. Hum. Percept. Perform. 20, 731-750.

Di Pellegrino, G., Fadiga, L., Fogassi, L., Gallese, V., and Rizzolatti, G. (1992). Understanding motor events: a neurophysiological study. Exp. Brain Res. 91, 176-180.

Donkers, F. C., and van Boxtel, G. J. (2004). The N2 in go/no-go tasks reflects conflict monitoring not response inhibition. Brain Cogn. 56, 165-176.

Folstein, J. R., and van Petten, C. (2008). Influence of cognitive control and mismatch on the N2 component of the ERP: a review. Psychophysiology 45, 152-170.

Forstmann, B. U., Jahfari, S., Scholte, H. S., Wolfensteller, U., van den Wildenberg, W. P. M., and Ridderinkhof, K. R. (2008a). Function and structure of the right inferior frontal cortex predict individual differences in response inhibition: a model-based approach. J. Neurosci. 28, 9790-9796.

Forstmann, B. U., van den Wildenberg, W. P.M., and Ridderinkhof, K. R. (2008b). Neural mechansims, temporal dynamics, and individual differences in interference control. J. Cogn. Neurosci. 20, 1854-1865.

Gallese, V., Fadiga, L., Fogassi, L., and Rizzolatti, G. (1996). Action recognition in the premotor cortex. Brain 119, 593-609.

Gallese, V., Keysers, C., and Rizzolatti, G. (2004). A unifying view of the basis of social cognition. Trends Cogn Sci 8, 396-403.

Gratton, G., Coles, M. G.H., and Donchin, E. (1992). Optimizing the use of information: strategic control of activation of responses. J. Exp. Psychol. 4, 480-506.
Hazeltine, E., Bunge, S. A., Scanlon, M. D., and Gabrieli, J. D. E. (2003). Material-dependent and materialindependent selection processes in the frontal and parietal lobes: an eventrelated fMRI investigation of response competition. Neuropsychologia 41 , 1208-1217.

Heil, M., Osman, A., Wiegelmann, J., Rolke, B., and Henninghausen, E. (2000). N200 in the Eriksen-task: inhibitory executive processes? J. Psychophysiol. 14, 218-255.

Hommel, B. (1994). Effects of irrelevant spatial S-R compatibility depend on stimulus complexity. Psychol. Res. 56, 179-184.

Hommel, B. (1995). Conflict versus misguided search as explanation of S-R correspondence effects. Acta Psychol. (Amst) 89, 37-51.

Hommel, B., Proctor, R. W., and Vu, K.P. L. (2004). A feature-integration account of sequential effects in the Simon task. Psychol. Res. 68, 1-17.

Hyvarinen, A., and Oja, E. (2000). Independent component analysis: algorithms and applications. Neural Netw 13, 411-413. 
Iacoboni, M., Molnar-Szakacs, I., Gallese, V., Buccino, G., Mazziotta, J. C., and Rizzolatti, G. (2005). Grasping the intentions of others with one's own mirror neuron system. PLoS Biol. 3, 529-535. doi: 10.1371/journal. pbio.0030079.

Iriarte, J., Urrestarazu, E., Valencia, M., Alegre, M., Malanda,A., Viteri, C., and Artieda, J. (2003). Independent component analysis as a tool to eliminate artifacts in EEG: a quantitative study. $J$. Clin. Neurophysiol. 20, 249-257.

Iverson, G. J.,Lee, M.D., and Wagenmakers, E.-J. (2008a). Prep misestimates the probability of replication. Psychon. Bull. Rev. 16, 424-429.

Iverson, G. J., Lee, M. D., Zhang, S., and Wagenmakers, E.-J. (2008b). Prep: an agony in five fits. J. Math. Psychol. 53, 195-202.

Jasper, H. H. (1958). The ten-twenty electrode system of the International Federation. Electroencephalogr. Clin. Neurophysiol. 10, 370-375.

Kerns, J. G. (2006). Anterior cingulate and prefrontal cortex activity in an FMRI study of trial-to-trial adjustments on the Simon task. Neuroimage 33, 399-405.

Kerns, J. G., Cohen, J. D., MacDonald, A. W. III, Cho, R.Y., Stenger, V. A., and Carter, C.S. (2004). Anterior cingulate conflict monitoring and adjustments in control. Science 303, 1023-1026.

Kohler, E., Keysers, C., Umilta, M. A., Fogassi, L., Gallese, V., and Rizzolatti, G. (2002). Hearing sounds, understanding actions: action representation in mirror neurons. Science 297, 846-848.

Kopp, B., Rist, F., and Mattler, U. (1996). N200 in the flanker task as a neurobehavioral tool for investigating executive control. Psychophysiology 33, 282-294.

Leuthold, H., and Schröter, H. (2006). Electrophysiological evidence for response priming and conflict regulation in the auditory Simon task. Brain Res. 1097, 167-180.

Loftus, G., and Masson, M. E. (1994). Using confidence intervals in withinsubject designs. Psychon. Bull. Rev. 1, 476-490.

Mansouri, F. A., Tanaka, K., and Buckley, M. J. (2009). Conflict-induced behavioural adjustment: a clue to the executive functions of the prefrontal cortex. Nat. Rev. Neurosci. 10, 141-152.

Mostofsky, S. H., and Simmonds, D. J. (2008). Response inhibition and response selection: two sides of the same coin. J. Cogn. Neurosci. 20, 751-761.

Nachev, P., Kennard, C., and Husain, M. (2008). Functional role of the supplementary and pre-supplementary motor areas. Nat. Rev. Neurosci. 9, 856-869.

Nieuwenhuis, S., Yeung, N., van den Wildenberg,W.P.M., andRidderinkhof, K. R. (2003). Electrophysiological correlates of anterior cingulate function in a go/no-go task: effects of response conflict and trial type frequency. $\operatorname{Cog} n$. Affec. Behav. Neurosci. 3, 17-26.

Notebaert, W., Soeters, E., and Melis, A. (2001). Sequential analysis of a Simon task - evidence for an attention-shift account. Psychol. Res. 65, 170-184.

Oldfield, R.C. (1971). The assessment and analysis of handedness: the Edinburgh inventory. Neuropsychologia 9, 97-113.

Pardo, J. V., Pardo, P. J., Janer, K. W., and Raichle, M. E. (1990). The anterior cingulate cortex mediates processing selection in the Stroop attentional conflict paradigm. Proc. Natl. Acad. Sci. 87, 256-259.

Phillips, M. L., Young, A. W., Senior, C., Brammer, M., Andrew, C., Calder, A. J., Bullmore, E. T., Perrett, D. I., Rowland, D., Williams, S. C. R., Gray, J. A., and David, A. S. (1997). A specific neural substrate for perceiving facial expressions of disgust. Nature 389, 495-498.

Ramnani, N., and Miall, R. C. (2004). A system in the human brain for predicting the actions of others. Nat. Neurosci. 7, 85-90.

Ridderinkhof, K. R., Ullsperger, M., Crone, E. A., and Nieuwenhuis, S. (2004a). The role of the medial frontal cortex in cognitive control. Science 306, 443-447.

Ridderinkhof, K. R., van den Wildenberg, W. P. M., Wijnen, J. G., and Burle, B. (2004b). Response inhibition in conflict tasks is revealed in delta plots. In Cognitive Neuroscience of Attention, M. Posner, ed., (New York, Guilford Press). pp. 369-377.

Rizzolatti, G. (2005). The mirror neuron system and its function in humans. Anat. Embryol. 210, 419-421.
Schuch, S., and Tipper, S. P. (2007). On observing another person's actions: influences of observed inhibition and errors. Percept. Psychophysiol. 69, 828-837.

Schumacher, E. H., Cole, M. W., and D'Esposito, M. (2007). Selection and maintenance of stimulus-response rules during preparation and performance of a spatial choice-reaction task. Brain Res. 1136, 77-87.

Simon, J. R., Acosta, E., Mewaldt, S. P., and Spiedel, C. R. (1976). The effect of an irrelevant directional cue on choice reaction time: duration of the phenomenon and its relation to stages of processing. Percept. Psychophys. 19, 16-22.

Simon, J. R., and Rudell, A. P. (1967) Auditory S-R compatibility: the effect of an irrelevant cue on information processing. J. Appl. Psychol. 51, 300-304.

Simon, R. J., and Wolf, J. D. (1963). Choice reaction times as a function of angular stimulus-response correspondence and age. Ergonomics 6, 99-105.

Small, D. M., Gregory, M. D., Mak, Y. E., Gitelman, D., Mesulam, M. M., and Parrish, T. (2003). Dissociation of neural representation of intensity and affective valuation in human gustation. Neuron 39, 701-711.

Stürmer, B., Leuthold, H., Soetens, E. and Schröter, H. (2002). Control over location-based response activation in the Simon task: behavioral and electrophysiological evidence. J. Exp. Psychol. 28, 1345-1363.

Tsai,C.C.,Kuo,W.J.,Hung,D.L., and Tzeng O. J. (2008). Action co-representation is tuned to other humans. J. Cogn. Neurosci. 20, 2015-2024.

Umiltá, M. A., Kohler, E., Gallese, V., Fogassi, L., Fadiga, L., Keysers, C., and Rizzolatti, G. (2001).'I know what you are doing' a neurophysiological study. Neuron 32, 91-101.

van Schie, H. T., Mars, R. B., Coles, M. G. H., and Bekkering, H. (2004). Modulation of activity in medial frontal and motor cortices during error observation. Nat. Neurosci. 7, 549-554.

Vigario, R. N. (1997). Extraction of ocular artefacts from EEG using independent component analysis. Electroencephalogr. Clin. Neurophysiol. 103, 395-404.
Wagenmakers, E-J. (2007). A practical solution to the pervasive problems of p values. Psychon. Bull. Rev. 14, 779-804.

Wetzels, R., Raaijmakers, J. G. W., Jakab, E., and Wagenmakers, E. J. (2009). How to quantify support for and against the null hypothesis: a flexible WinBUGS implementation of a default Bayesian t-test. Psychon. Bull. Rev. 16, 752-760.

Whiten, A., and Byrne, R. W. (1997). Machiavellian Intelligence II: Extensions and Evaluations. Cambridge, Cambridge University Press.

Wijnen, J. G., and Ridderinkhof, K. R. (2009). Contextual modulation of oculomotor control reflected in N2 and saccade reaction time distributions. Psychophysiol. 46, 1033-1042.

Wühr, P., and Ansorge, U. (2005). Exploring trial-by-trial modulations of the Simon effect. Q. J. Exp. Psychol. A 58, 705-731.

Yeung, N., Botvinick, M. M., and Cohen, J. D. (2004). The neural basis of error detection: conflict monitoring and the error-related negativity. Psychol. Rev. 4, 931-959.

Conflict of Interest Statement: The authors declare that the research was conducted in the absence of any commercial or financial relationships that could be construed as a potential conflict of interest.

Received: 03 June 2009; paper pending published: 25 June 2009; accepted: 14 November 2009; published online: 04 December 2009.

Citation: Winkel J, Wijnen JG, Ridderinkhof KR, Groen IIA, Derrfuss J, Danielmeier C and Forstmann BU (2009) Your conflict matters to me! Behavioral and neural manifestations of control adjustment after self-experienced and observed decisionconflict. Front. Hum. Neurosci. 3:57. doi: 10.3389/neuro.09.057.2009

Copyright (c) 2009 Winkel, Wijnen, Ridderinkhof, Groen, Derrfuss, Danielmeier and Forstmann. This is an open-access article subject to an exclusive license agreement between the authors and the Frontiers Research Foundation, which permits unrestricted use, distribution, and reproduction in any medium, provided the original authors and source are credited. 